\title{
Perception of the duration of sleep intervals as a function of EEG sleep stage
}

\author{
V. R. CARLSON \\ National Institute of Mental Health, Bethesda, Maryland 20014 \\ I. FEINBERG \\ Veterans Administration Hospital, San Francisco, California 94121 \\ and University of California at San Francisco, San Francisco, California 94143 \\ and \\ D. R. GOODENOUGH \\ Educational Testing Service, Princeton, New Jersey 08540

\begin{abstract}
Subjects were able to estimate with relative accuracy the durations of sleep intervals made up predominantly of EEG stage 1-REM but not of delta. Successful estimation required recall of a stimulus marking the beginning of an interval, but did not depend on time awake or on the EEG stage just prior to awakening. These results are consistent with the proposition that the passage of time is registered as part of the ongoing mentation of REM sleep. Correlation of time estimates with elapsed time for REM periods but not for delta periods makes comparison of the mean time estimates for these two conditions equivocal.
\end{abstract}

Human subjects can judge elapsed time over a period of sleep with better than chance accuracy (Boring \& Boring, 1917; Danilin \& Latash, 1972; Lavie \& Webb, 1975; Lewis, 1969; Noble \& Lundie, 1974; Tart, 1970; Zung \& Wilson, 1971). This statistically significant accuracy, however, does not necessarily reflect a genuine ability to perceive the passage of time during sleep. If, for example, one feels more rested after a longer period of sleep, one may judge the time asleep as longer, but the judgment may reflect the feeling of restedness rather than subjective elapsed time. Or, for a longer period of sleep, a greater number of transient periods of waking may occur and the judgment may be based on the amount of waking that took place. If, however, the passage of time is registered during sleep, one might expect time estimates to differ for different stages of the sleep EEG, because of putative differences among these stages in ongoing mentation and neurophysiologic activity (Berger, 1969a; Snyder \& Scott, 1972). Estimations of the durations of sleep periods have, in fact, been reported to be related to sleep stage (Danilin \& Latash, 1972) ${ }^{1}$ but have also been reported not to be related to sleep stage (Zung \& Wilson, 1971).

The conflicting evidence may have arisen in part because mean errors in time estimates for different stages were being compared. Mean comparisons do not tell us whether variations in the time estimates

Requests for reprints should be sent to V. R. Carlson, Laboratory of Psychology and Psychopathology, National Institute of Mental Health, Bethesda, Maryland 20014. are related to variations in elapsed time or not. If no such relationship exists, the estimates may depend on secondary factors peculiar to the particular circumstances of the experiment. If there is a relationship between time estimates and elapsed time for one condition but not for another, the meaningfulness of a comparison between the means for the two conditions is uncertain. The basic questions that need to be answered are whether there is a correlation between estimated and elapsed times over varying periods of sleep and whether the magnitude of this correlation differs for different sleep stages. The present study was an attempt to answer these two questions.

\section{METHOD}

\section{Subjects}

Ten normal male volunteers, 19-24 years old, were paid to participate in the study. Four had had prior experience as subjects in sleep research. The distributions of measures for these four subjects substantially overlapped the distributions for the other six, and no further comparison of the two groups was undertaken.

\section{Procedure}

Subjects slept in a light-, sound-, and temperature-controlled room which permitted no extraneous time cues. They were awakened and presented with a stimulus picture and descriptive phrase and allowed to go back to sleep. Later they were awakened again and asked to recall the previously presented stimulus and to estimate the time that had elapsed since its presentation. If the subject could not remember the picture, he was asked nevertheless to make the best time estimate he could. Each subject estimated five (nonconcurrent) time intervals per night.

The subject was awakened by the ringing of a telephone 
mounted on the wall at the side of the bed. He was required to lift the phone off the hook as quickly as he could, say "hello," and state the descriptive phrase and his time estimate for the preceding interval or look at a current picture and repeat its descriptive phrase, and hang up the phone.

\section{Interval Onset Stimuli}

The stimuli identifying the beginnings of the time intervals to be estimated were pictures photographed from magazines, mounted on slides, and projected by remote control onto a rear-projection screen at the foot of the bed. Because pilot studies had shown a high rate of forgetting, the pictures were selected to be unusual and memorable (e.g., a prize fighter wearing pink tights). The pictures were assigned at random, and the same picture was not shown more than once to each subject. The descriptive phrase was communicated to the subject through a speaker located in the ceiling above the subject's head. A recognizable description of the picture, not necessarily an exact repetition of the phrase, was taken to indicate recall of the picture. Intervals for which the picture presented at the beginning of the interval was correctly recalled are referred to as "onset-recalled" intervals. Intervals for which the picture was not recalled or was incorrectly recalled are referred to as "onset-nonrecalled" intervals.

\section{Sleep Intervals}

The EEG and electrooculogram were monitored continuously, as described in Feinberg, Koresko, and Heller (1967). We attempted to distribute the awakenings and longer and shorter time intervals as evenly as possible between earlier and later occurrences of EEG stages 1-REM (rapid eye movement), 2, and delta (stages 3 and 4 combined). In order to obtain reasonably well-distributed intervals, it was necessary to test four of the subjects for 7 (nonconsecutive) nights, four for 8 nights, and two for 9 nights. Turning on the projector for presentation of the picture was recorded as the beginning of an interval, and the subject's picking up of the telephone was recorded as the end of the interval. Scoring of the EEG and electrooculogram records was done without knowledge of the subjects' time estimates or recall of the onset stimuli.

\section{RESULTS AND DISCUSSION}

The actual time intervals varied from $10 \mathrm{~min}$ to $2 \mathrm{~h}$ (mean $=38.7 \mathrm{~min}, \mathrm{SD}=18.6)$. Analysis of the variations in these values revealed no statistically significant bias in the presentation of the intervals with respect to time of night or EEG stage; i.e., shorter and longer intervals occurred about equally often for the first through fifth judgment intervals and for each EEG stage. The subjects' time estimates ranged from $5 \mathrm{~min}$ to $3 \mathrm{~h}$ (mean $=61.6 \mathrm{~min}, \mathrm{SD}=39.6$ ). Sixty-seven percent of the interval onset stimuli were recalled, varying from $33 \%$ to $89 \%$ among subjects, and the EEG distribution differed for onset-recalled vs. onset-nonrecalled intervals (left side of Table 1) $[\mathrm{F}(3,27)=5.88, \mathrm{p}<.005]$. The onset-recalled intervals were characterized by greater time awake and less stage delta, consistent with previous findings and interpretations on the retention of words presented before falling asleep (Goodenough, Sapan, Cohen, Portnoff, \& Shapiro, 1971). Principally, most of the time awake occurred after presentation of the stimulus and before falling asleep, when greater time awake would allow for more rehearsal of the stimulus and consequently better recall.

When a subject could not remember the beginning of an interval, it would seem reasonable if he judged the time back to some earlier period that he did remember, perhaps even back to the time of retiring. Consequently, the estimates for onset-nonrecalled intervals might tend to be greater than those for onsetrecalled intervals. Such a tendency did seem to occur for seven subjects, but not for three, and the overall difference in mean time estimate for onset-recalled vs. -nonrecalled intervals was not statistically significant.

Our main analysis was in terms of the correlation of the variations in the individual subjects' time estimates with the corresponding actual variations in the time intervals. Product-moment correlation coefficients were computed for each subject for each condition, converted to Fisher's $\mathrm{z}$ values, and averaged over subjects, weighting each $z$ value by the number of observations upon which the correlation was based. The reported averages are the productmoment correlation coefficients corresponding to the mean $\mathrm{z}$ values.

If, in judging time, the subjects were merely reflecting their subjective state at the time of making the judgment, then it should have made no difference whether the stimulus presented at the beginning of the interval was remembered or not. It did clearly make a difference, however. For the time intervals whose onset stimuli were recalled, the average correlation of the time estimates with the actual elapsed times was significant; for the intervals whose onset stimuli were not recalled, the correlation was zero (right side of Table 1). Moreover, although the onsetrecalled intervals were characterized by greater waking time, the time estimates were not correlated with time

Table 1

Comparison of Intervals for Which Onset Stimulus Was Recalled with Intervals for Which Onset Stimulus Was Not Recalled

EEG Percent of Interval

\begin{tabular}{|c|c|c|c|c|c|c|c|c|c|c|c|c|}
\hline \multirow{3}{*}{$\begin{array}{c}\text { Interval } \\
\text { Onset }\end{array}$} & & \multirow{2}{*}{\multicolumn{2}{|c|}{ Time Awake }} & & \\
\hline & \multicolumn{2}{|c|}{ Awake } & \multicolumn{2}{|c|}{ 1-REM } & \multicolumn{2}{|c|}{ Stage 2} & \multicolumn{2}{|c|}{ Delta } & & & \multicolumn{2}{|c|}{ Elapsed Time } \\
\hline & Mean & SE & Mean & SE & Mean & SE. & Mean & SE & Mean & SE & Mean & SE \\
\hline Recalled & 21.9 & .9 & 18.7 & 1.0 & 42.5 & 1.4 & 16.9 & 1.4 & .02 & .06 & $.41^{*}$ & .09 \\
\hline Not Recalled & 16.1 & 1.6 & 22.5 & 2.6 & 36.9 & 3.0 & 24.5 & 2.0 & -.01 & .09 & .04 & .14 \\
\hline
\end{tabular}

Note $-N=10 . \quad{ }^{*}$ Corresponding $z$ value significantly different from zero $[t(9)=4.71, p<.005]$. 
awake. Thus, it appears that subjects have some ability to perceive the passage of time over a sleep interval independently of time spent awake, provided they can recall the beginning of the interval being judged.

The onset-recalled intervals were further analyzed according to the EEG stage predominating during the interval. The upper part of Table 2 shows the average composition of these intervals with respect to percentages of the EEG stages. Awakenings were deliberately distributed to try to maximize and equalize the predominance of the three stages, and the distribution in Table 2 indicates that this goal was reasonably well achieved.

The lower part of Table 2 shows the average correlations between the time estimates and elapsed times for the onset-recalled intervals, separately for intervals over which the EEG was predominantly stage 1-REM, 2, or delta. The relationship was highly significant for 1-REM and not significant for delta. The result for stage 2 was intermediate, admitting two possibilities. The true correlation for stage 2 might be high but reduced by the presence of some stage delta. Alternatively, the true correlation for stage 2 might be low but increased by the presence of some stage 1-REM. Partialling out the correlations with amounts of 1-REM and delta resulted in a nonsignificant correlation for stage 2 [average $r_{p}=$ $.35, \mathrm{SE}=.20, \mathrm{t}(9)=1.79, \mathrm{p}>.10]$. We therefore conclude that the ability to perceive elapsed time during sleep has been demonstrated for intervals made up predominantly of stage 1-REM, but not for intervals made up predominantly of stage 2 or delta. ${ }^{2}$

For most of the predominantly stage 1-REM intervals, the subjects were awakened from stage 1-REM. Consequently, the significant correlation between the time estimates and 1-REM intervals might have depended upon the sleep stage at the time of arousal rather than upon the predominant EEG stage during

Table 2

Average Within-Intervals EEG Percentages and Average Correlations of Time Estimates with Elapsed Times (Intervals for Which Onset Stimuli Were Recalled)

\begin{tabular}{|c|c|c|c|c|c|c|}
\hline \multirow[b]{3}{*}{ Measure } & \multicolumn{6}{|c|}{ Predominant EEG Stage } \\
\hline & \multicolumn{2}{|c|}{ 1-REM } & \multicolumn{2}{|c|}{ Stage 2} & \multicolumn{2}{|c|}{ Delta } \\
\hline & Mean & SE & Mean & SE & Mean & SE \\
\hline \multicolumn{7}{|l|}{ Percentage } \\
\hline 1-REM & 55 & 1.5 & 5 & 1.1 & 1 & .9 \\
\hline Stage 2 & 19 & 2.8 & 62 & 1.3 & 29 & 1.8 \\
\hline Delta & 2 & .9 & 10 & 1.5 & 54 & 1.5 \\
\hline Awake & 24 & 1.8 & 23 & 1.2 & 16 & 1.6 \\
\hline Correlation & $.60 *$ & .13 & $.33^{* *}$ & .12 & $.30 \dagger$ & .24 \\
\hline
\end{tabular}

Note $-N=10$. Correlational analysis was performed on Fisher's $z$ values. $\quad{ }^{*} t(9)=5.29, p<.001 . \quad{ }^{* *} t(9)=2.70, p=.025$. $f_{t}(9)=1.27$, n.s. the interval. We lacked sufficient data to compute the correlations separately for individual subjects. However, there were 32 instances in which the onset stimulus was recalled and awakening was from 1 -REM but the interval was not predominantly 1-REM. Computing the deviations of these time estimates from the individual subject means and treating them as though they all were obtained from a single subject resulted in a nonsignificant correlation of .19 with the time values $[F(1,30)=1.14]$. Performing the same test for the 62 instances in which the awakening was from stage 1-REM and the interval was predominantly $1-$ REM, the correlation was .58 $[\mathrm{F}(1,60)=30.6, \mathrm{p}<.001]$.

Thus, predominance of stage 1-REM during the sleep periods, rather than awakening from this stage at the end of the periods, was required for the significant correlation between the estimated and actual durations. This interpretation is further supported by the lack of relationship of the awakening EEG to arousal at a predesignated time (Zung \& Wilson, 1971) or to judgments of short time intervals immediately after awakening (Carlson \& Feinberg, 1976).

These correlational results fit well with a conclusion of Dement and Kleitman: The duration of dream activity occurring during REM periods corresponds approximately to the time that would be required for the dreamed events actually to take place (Dement \& Kleitman, 1957; Kleitman, 1963, p. 101). If the passage of time is registered as it would be if the events were actually occurring, it follows that the impiession of elapsed time would be related to real elapsed time. It has not been established, however, that the correspondence between subjective dream duration and real time is better for dreams occurring during REM sleep than for dreams occurring during non-REM sleep. The question is greatly complicated by the possibility that such correspondence may differ for dreams of differing content (Moiseeva, 1975) and by the fact that it is difficult to determine whether the actual kind and amount of dreaming, or the later recall of it, varies among the sleep stages (Berger, 1969b).

Danilin and Latash (1972) report differences in time estimation dependent on sleep cycle as well as on sleep stage. Again, using the deviations from the individual subject means and disregarding subjects, our correlations between the time estimates and elapsed times for the first through the fifth intervals judged during the night were $.51, .60, .86, .61$, and .59 , respectively, for intervals for which the onset stimuli were recalled and the predominant EEG was 1-REM. The corresponding correlations for predominantly non-REM intervals were $-.01, .21, .38, .46$, and .22 . While these values cannot be demonstrated to differ among themselves, because no appropriate statis- 
tical test is available, the apparent trends are consistent with possible time-of-night effects.

The finding that judged time is correlated with elapsed time under some conditions but not others may help to account for empirical inconsistencies in mean time estimates. In our data for which the beginnings of the intervals were recalled, the mean estimate for delta periods $(77.8 \mathrm{~min}, \mathrm{SE}=11.1)$ was greater than that for REM periods $(60.1 \mathrm{~min}, \mathrm{SE}=$ 7.6). This is the reverse of the mean effects found by Danilin and Latash (1972). But the difference between these means is not logically generalizable. If we had presented longer intervals, everything else in the situation remaining the same, the average judgment for REM would be expected to increase, because these judgments were correlated with real time. The judgments for delta would not be expected to change, because they were not correlated with real time. The mean estimate for REM would then be the same as, or greater than, the mean for delta. The mean difference would thus depend upon the particular intervals chosen by the experimenter, rather than upon any general tendency for REM periods to be experienced as longer or shorter than delta periods.

\section{REFERENCES}

Berger, R. J. Physiological characteristics of sleep. In A. Kales (Ed.), Sleep physiology and pathology. Philadelphia: Lippincott, 1969. (a)

Berger, R. J. The sleep and dream cycle. In A. Kales (Ed.), Sleep physiology and pathology. Philadelphia: Lippincott, 1969. (b)

Boring, L. D., \& Boring, E. G. Temporal judgments after sleep. In Studies in psychology. Worcester: Wilson, 1917.

Carlson, V. R., \& Feinberg, I. Judgments of short time intervals following awakenings from different EEG stages of sleep. Physiological Psychology, 1976, 4, 341-345.

Danilin, V. P., \& Latash, L. P. [Subjective judgments of the duration of periods of night sleep upon awakening during different sleep stages, phases, and cycles.] Doklady Akademii Nauk SSSR, 1972, 204, 748-751.

Dement, W., \& Kleitman, N. The relation of eye movements during sleep to dream activity: An objective method for the study of dreaming. Journal of Experimental Psychology, 1957, 53, 339-346.
Feinberg, I., Koresko, R. L., \& Heller, N. EEG sleep patterns. as a function of normal and pathological aging in man. Journal of Psychiatric Research, 1967, 5, 107-144.

Goodenough, D. R., Sapan, J., Cohen, H., Portnoff, G., \& ShapIRo, A. Some experiments concerning the effects of sleep on memory. Psychophysiology, 1971, 8, 749-762.

Kleitman, N. Sleep and wakefulness (rev. ed.). Chicago: University of Chicago Press, 1963.

Latash, L. P., \& Danilin, V. P. Subjective estimation of the duration of time periods in night sleep. Nature New Biology, 1972, 236, 94-95.

Latash, L. P., Danilin, V. P., Manov, G. A., \& Rait, M. L. On the functional interrelations between delta sleep and REM sleep. In P. Levin \& W. P. Koella (Eds.), Sleep 1974. 2nd European Congress on Sleep Research, Rome 1974. Basel: Karger, 1975.

MoIseEVA, N. I. The characteristics of EEG activity and the subjective estimation of time during dreams of different structure. Electroencephalography and Clinical Neurophysiology, 1975, 38, 569-577.

LAVIE, P., \& WebB, W. B. Time estimates in a long-term time-free environment. American Journal of Psychology, 1975, 88, 177-186.

LEwIs, S. A. Subjective estimates of sleep: An EEG evaluation. British Journal of Psychology, 1969, 60, 203-208.

Noble, W. G., \& Lundie, R. E. Temporal discrimination of short intervals of dreamless sleep. Perceptual and Motor Skills, 1974, 38, 445-446.

SNYDER, F., \& ScotT, J. The psychophysiology of sleep. In N. S. Greenfield \& R. A. Sternback (Eds.), Handbook of psychophysiology. New York: Holt, Rinehart, \& Winston, 1972.

TART, C. T. Waking from sleep at a preselected time. Journal of the American Society of Psychosomatic Dentistry and Medicine, 1970, 17, 3-16.

Zung, W. W. K., \& Wilson, W. P. Time estimation during sleep. Biological Psychiatry, 1971, 3, 159-164.

\section{NOTES}

1. Reports of this research in English are contained in Latash and Danilin (1972) and Latash, Danilin, Manov, and Rait (1975).

2. A stronger argument would have been possible for the dependence of subjective duration on the specific type of EEG activity, if the time estimates had been correlated with the amount of one of the EEG stages independently of the total duration of the interval. Such was not the case. Partialling out the durations of the intervals resulted in nonsignificant correlations between the time estimates and the EEG durations for all three stages.

(Received for publication February 28, 1978; revision accepted September $15,1978$. 